\title{
Prosaposin in seminal plasma on the day of oocyte retrieval is associated with normal fertilization and embryo development in in vitro fertilization cycles
}

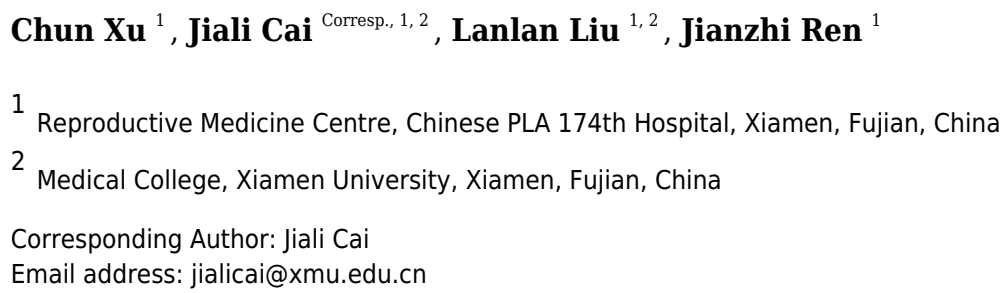

The prospective study including 166 participants aims to evaluate the association between seminal prosaposin and the outcomes of in vitro fertilization (IVF) cycles in humans. Generalized linear model (GLM) was used to analyze the associations between seminal prosaposin concentrations and normal fertilization rates and good embryos proportion. Generalized estimating equation (GEE) was used to evaluate the association between embryo parameters and the prosaposin concentrations. Each model was adjusted for age of the couples, female basal FSH, AFC and BMI, starting dose and oocyte yield of IVF cycles and smoker. GLM models suggested that prosaposin was significantly associated with fertilization rate $(P=0.005)$ and good embryo proportion $(P=0.038)$ while none of the semen parameters (sperm concentration, motility, progressive motility, normal morphology rate, postwash sperm concentration and motility) was significantly associated with the parameters in the cohort. Using GEE, it is also shown that prosaposin was positively associated with the occurrence of early cleavage and negatively associated with uneven cleavage pattern on day 3 . In both the overall population and the normozoospermia patients, the prosaposin was significantly associated with pregnancy with adjustment with covariates. In conclusion, our data suggested that seminal prosaposin concentration could provide more information regarding normal fertilization and embryo development in IVF than traditional semen parameters. 
1 Prosaposin in seminal plasma on the day of oocyte retrieval is associated with normal

2 fertilization and embryo development in in vitro fertilization cycles.

3

4 Chun Xu, M.D. ${ }^{1}$, Jiali Cai, Ph.D. 1,2,*, Lanlan Liu, Ph.D. ${ }^{1,2}$, Jianzhi Ren, M.D. ${ }^{1}$

$5 \quad{ }^{1}$ Reproductive Medicine Centre, Chinese PLA 174th Hospital, Xiamen, Fujian, China.

$6 \quad 2$ Medical College, Xiamen University, Xiamen, China

8 *Corresponding Author:

9 Jiali Cai

10 Wenyuan road, Xiamen, Fujian, 361002, China.

11 Email address: Jialicai@xmu.edu.cn 
18 Abstract

19 The prospective study including 166 participants aims to evaluate the association between

20 seminal prosaposin and the outcomes of in vitro fertilization (IVF) cycles in humans.

21 Generalized linear model (GLM) was used to analyze the associations between seminal

22 prosaposin concentrations and normal fertilization rates and good embryos proportion.

23 Generalized estimating equation (GEE) was used to evaluate the association between embryo

24 parameters and the prosaposin concentrations. Each model was adjusted for age of the couples,

25 female basal FSH, AFC and BMI, starting dose and oocyte yield of IVF cycles and smoker.

26 GLM models suggested that prosaposin was significantly associated with fertilization rate

$27(\mathrm{P}=0.005)$ and good embryo proportion $(\mathrm{P}=0.038)$ while none of the semen parameters (sperm

28 concentration, motility, progressive motility, normal morphology rate, postwash sperm

29 concentration and motility) was significantly associated with the parameters in the cohort. Using

30 GEE, it is also shown that prosaposin was positively associated with the occurrence of early

31 cleavage and negatively associated with uneven cleavage pattern on day 3 . In both the overall

32 population and the normozoospermia patients, the prosaposin was significantly associated with

33 pregnancy with adjustment with covariates. In conclusion, our data suggested that seminal

34 prosaposin concentration could provide more information regarding normal fertilization and

35 embryo development in IVF than traditional semen parameters.

36 


\section{Introduction}

Semen quality, as measured according to a widely criteria established by world health organization (WHO) is perhaps the most important marker for male infertility potential in clinical use at the point of time(Sakkas et al. 2015; WHO 2010). The sperm number and motility are not only the key determining factors for the "numbers game" to achieve the necessary tens to hundreds of sperm in the ampulla (Sakkas et al. 2015) but also the indicators for the health status of male reproductive system itself (Choy \& Eisenberg 2018).

In the era of ART, in vitro manipulation of gametes allows bypassing of the natural selection barrier for the spermatozoon in the female reproductive tract, such as hostile vaginal $\mathrm{pH}$, barrier of cervical mucus and immune response in the tract, and thus reduces the importance of the "numbers game" to reach the oocytes (Sakkas et al. 2015). Conflicting results are obtained from studies regarding the association between male semen quality and IVF outcomes. A recent study suggested that advancing male age, elevated BMI or poor sperm quality is not associated with outcomes in frozen donor oocyte IVF cycles (Capelouto et al. 2018). It suggested that semen quality alone may not yield sufficient information regarding the potential of male fertility as soon as the spermatozoon reaches the oocytes.

Cumulative studies have investigated and evaluated various additional seminal/sperm markers beyond traditional seminal quality, among which the abundant tissue-specific proteins within the seminal plasma provide a rich source of potential candidates (Bieniek et al. 2016; Cao et al. 2018). Proteomic and biomarker discovery technologies have linked lists of proteins to male infertility etiologies, exposure and life styles (Intasqui et al. 2015). However, few proteins 
60

61 fertilization event and subsequent embryo development.

62

63

64 between seminal proteins and reproductive outcomes culture are used as secondary outcomes.

among the lists has been associated with the functions of the reproductive process, such as

$$
\text { Prosaposin is known as a lysosomal protein found in Sertoli cells and the lumen of the }
$$
seminiferous tubules and epididymis of mammals (Morales et al. 1998) as well as a secretary protein identified in the seminal proteome for both men and animals (Codognoto et al. 2018; Sharma et al. 2013; Viana et al. 2018). In bulls, fertility rank is positively associated seminal concentration of prosaposin(Viana et al. 2018). In vitro studies showed that the protein contributes to the sperm-oocyte binding, fertilization and embryo development in several species (Amann et al. 1999a; Amann et al. 1999b; Amann et al. 1999c; Hammerstedt et al. 2001; Magargee et al. 2000). The physiological role of the protein may suggest a functional link IVF cycles may provide an ideal model to observe the association between postulated markers and events following sperm-oocyte interaction, such as fertilization and embryo cleavage. The present study aims to evaluate the association between seminal prosaposin concentration and normal fertilization. Additionally, embryo cleavage patterns during in vitro 


\section{Materials and Methods}

Institutional review board approval for this study was obtained from the Ethical Committee of Medical College Xiamen University. All the subjects enrolled in this study were given written formal consent before participation. No clinical trial registry was necessary because the study did not involve any type of intervention.

\section{Participants}

Participants were recruited between Jan 2013 and June 2016. To minimize the confounding from female participants, we included only patients receiving conventional IVF treatment for the first time, undergoing conventional long agonist for ovarian stimulation. Female participants were with good physical and mental health, aged $<35$ years; regular menstrual cycles ranging from 25 to 35 days; $\mathrm{BMI}<28 \mathrm{~kg} / \mathrm{m}^{2}$; normal basal serum $\mathrm{FSH}(\leq 10 \mathrm{mIU} / \mathrm{ml})$ and estradiol $\left(\mathrm{E}_{2}\right)(\leq 75$ $\mathrm{pg} / \mathrm{ml}$ ). No sign of male reproductive tract infection was detected in the male participants. The exclusion criteria were: patients with endometriosis or PCOS, patients with suboptimal ovarian response (oocyte yield $<5$ ) and patients with sign of OHSS.

\section{Ovarian stimulation}

All patients received the same regimen using depot GnRH agonist (Ren et al. 2014). Patients received 2-3 ampoules (150-225 IU) gonadotrophin per day during the gonadotrophin stimulation. The starting dose was adjusted according to patients`age, AFC and BMI. Physicians triggered oocyte maturation using 5000-10000 IU human chorionic gonadotrophin (hCG; Lizhu Pharma, China) as soon as ultrasonography revealed at least one follicle measuring $\geq 18 \mathrm{~mm}$ in mean diameter. Oocyte retrieval was scheduled 34 to $36 \mathrm{hr}$ after triggering. 


\section{Semen preparation}

Semen parameters of male counterparts were evaluated according to WHO criteria.

On the day of oocyte retrieval, semen was produced by masturbation and motile spermatozoa were prepared by centrifugal fractionation $(350 \mathrm{G}, 10 \mathrm{~min})$ using sperm isolation medium (Isolate, Irvine Scientific, CA). Resulting spermatozoa was washed $(250 \mathrm{G}, 5 \mathrm{~min})$ in gamete buffer (KSIGB-50, Cook, Australia) and incubated in $37^{\circ} \mathrm{C}$ until insemination.

Semen samples $(100 \mu \mathrm{l})$ for prosaposin determination were collected before centrifugal fractionation. The spermatozoa were removed following high speed centrifugation (14000G, 10min). The seminal plasma was stored at $-80{ }^{\circ} \mathrm{C}$ until use.

\section{Embryo culture and assessment}

Oocytes were inseminated $4 \mathrm{hr}$ after collection. Pronuclei (PN) were identified $18 \mathrm{hr}$ later. All embryos were cultured in traditional incubators (C200, Labotech, Germany) at $37{ }^{\circ} \mathrm{C}, 6 \% \mathrm{CO}_{2}$, $5 \% \mathrm{O}_{2}$. Occurrence of early cleavage event was observed at $27 \mathrm{hr}$ post insemination. Day 3 embryos were graded based on numbers of embryo blastomere, fragmentation, and symmetry. Grade 1 and grade 2 embryos were considered as high quality embryos, grade 1, grade 2, and grade 3 embryos were considered as available embryos. Embryo transfer procedure and pregnancy determinant were described in our previous research.

\section{Determination of prosaposin}

Prosaposin was determined using an ELISA kit (Uscn Life Science Inc., Wuhan, China) according to the manufacturer's instructions. All samples were thawed and diluted five folds in PBS. The color change of the substrate is measured spectrophotometrically at a wavelength of 
$119450 \mathrm{~nm}$. The concentration of prosaposin in the samples is then determined by comparing the

120 O.D. of the samples to the standard curve. The lower detection limit of the analysis is less than

$1218.2 \mathrm{pg} / \mathrm{ml}$

122 Statistical analysis

123 The primary outcome of the study was normal fertilization rate. Normal fertilization rate rather

124 than total fertilization rate was used because it is a key performance indicator for IVF procedure

125 (ESHRE Special Interest Group of Embryology and Alpha Scientists in Reproductive Medicine,

126 2017) and is more relevant to the outcome. The secondary outcomes were the parameters

127 associated with embryo development following fertilization, including good embryo proportion,

128 occurrence of early event on day 1, fragmentation, cleavage speed and cleavage pattern on day 3.

129 Normal fertilization rate was defined as the proportion of $2 \mathrm{PN}$ or $2 \mathrm{~PB}$ of cumulus-oocyte

130 complexes inseminated (ESHRE Special Interest Group of Embryology and Alpha Scientists in

131 Reproductive Medicine, 2017). Good embryo proportion was the proportion of good embryos

132 among normally fertilized oocytes.

133 Generalized linear model was used to evaluate the association between prosaposin concentration

134 and good embryos proportion. The model was adjusted for age of the couples, female basal FSH,

135 AFC and BMI, starting dose and oocyte yield of IVF cycles and male smoker.

136 Generalized estimating equation was used to analyze the association between prosaposin

137 concentrations and embryo parameters (fragmentation $>10 \%$, early cleavage, fast cleavage, slow

138 cleavage, on time cleavage, unsynchronized cleavage and uneven cleavage). Embryos from the

139 same cycle were treated as dependent samples in the analysis.

Peer) reviewing PDF | (2019:07:39370:1:1:NEW 21 Oct 2019) 
140 Traditional semen parameters (sperm concentration, motility, progressive motility, normal

141 morphology rate, postwash sperm concentration and motility) were also associated with the

142 outcomes in the same manner.

143 Multivariate model was also used to detect the association between prosaposin and pregnancy.

144 Beside the covariate aforementioned, the model was also adjusted for endometrial thickness,

145 stage of embryo transferred (cleavage vs blastocyst), number of embryos transferred and distance

146 from transfer catheter tip to fundal.

147 Correlation between prosaposin concentrations and semen parameters were calculated according

148 to spearmen correlation coefficient.

149 Data analysis was performed using SPSS Version 19.0 statistical software (IBM, Armonk, NY).

\section{Results}

151 A total of 166 couples were involved in the study. As demonstrated in Table 1, female

152 counterparts were normal responders with a median oocyte yield of 11 . The median age of the

153 female patient was 31 years. None of the selected patients was with endometriosis, PCOS or

154 other diagnosed endocrine dysfunction. Among male counterparts, the median age was 32 years.

155 More than half of them $(n=87,52.4 \%)$ were with normozoospermia according to WHO criteria.

156 Others were with asthenozoospermia $(n=54)$, teratozoospermia $(n=12)$, asthenoteratozoospermia

$157(n=9), \quad$ oligoasthenozoospermia $\quad(n=2), \quad$ oligoasthenoteratozoospermia $\quad(n=1) \quad$ or

158 oligozoospermia $(n=1)$. The median prosaposin concentration in the seminal plasma was 73.12

$159 \mathrm{ng} / \mathrm{ml}$. The prosaposin concentration significantly correlated with progressive motility and

160 postwash sperm motility but not sperm concentration or morphology (Table 2). 
161 In multivariate analyses, the concentrations of seminal prosaposin were positively associated

162 with normal fertilization rate $(\mathrm{P}<0.01)$. The regression coefficient indicated that $\mathrm{per} \mathrm{ng} / \mathrm{ml}$

163 increase in seminal prosaposin would lead to $0.066 \%$ increase in normal fertilization rate after

164 adjustment of confounding factors. On the other hand, however, none of the traditional semen

165 parameters, such as sperm concentration, motility and normal morphology rate was significantly

166 associated with normal fertilization rate (Table 3). To test the robustness of the association, we

167 carried out a sensitivity analysis in a subgroup of patients with normozoospermia $(\mathrm{n}=87)$.

168 Although the $\mathrm{P}$ value (0.042) was increased due to the small sample size, the result was

169 consistent with that in the total population (Table 3).

170 To compare the ability of seminal prosaposin and standard semen parameters in predicting low

171 fertilization events (normal fertilization rate $<50 \%$ ), receiver operating characteristic (ROC)

172 curves was used to determine which cutoff would provide the best trade-off between sensitivity

173 and specificity and AUC for each predictors were qualified (Table 4). It is shown that in both the

174 overall population and the normozoospermia men, prosaposin showed limited discriminating

175 capacity to predict the cycles with low fertilization, where either the sperm concentration nor the

176 sperm motility showed any discriminating capacity ( $\mathrm{AUC}<0.6, \mathrm{P}>0.05$ ).

177 In Table 5, we further associated individual embryo parameter with prosaposin using GEE and

178 found that prosaposin concentration was significantly associated with the occurrence of early

179 cleavage on day 1 and uneven cleavage pattern on day3 (Table 5). On the other hand, the sperm

180 motility was significantly associated with early cleavage and postwash sperm motility was

181 negatively associated with fast cleaving of day 3 embryos.

Peer) reviewing PDF | (2019:07:39370:1:1:NEW 21 Oct 2019) 
182 We also explored the association between seminal prosaposin and pregnancy following embryo

183 transfer (Table 6). With adjustment of semen volume, the prosaposin concentration was

184 significantly associated with pregnancy. In univariate model, however, prosaposin was not

185 significantly associated with pregnancy in either overall population (OR 1.004, 95\% CI: 0.998-

186 1.004) or Normozoospermic men (OR 1.006, 95\%CI: 0.998-1.014). 


\section{Discussion}

Although the detail is not clear, the role of prosaposin in male fertilization and spermatozoa-oocyte interaction has been revealed in several species (Amann et al. 1999a; Amann et al. 1999b; Amann et al. 1999c; Hammerstedt et al. 2001; Magargee et al. 2000). Conservation of evolution may suggest the importance of the protein in male reproductive process. However, it is still not known whether there is any clinical importance of this protein among patients receiving infertility treatment. The present study is adding to existing knowledge by demonstrating the role of seminal prosaposin in predicting the fertilization, embryo development and pregnancy in patients receiving IVF. The data suggested that prosaposin concentration in semen not only had moderate predicting value in low fertilization event but also significantly correlated to embryo development and pregnancy following fertilization. Because seminal plasma is removed during preparation of spermatozoon in IVF procedures, the prosaposin in seminal plasma may not be directly involved in the sperm-oocyte interaction. It is possible that the concentration of prosaposin reflects the health status of reproductive system. As shown in our previous study, the prosaposin associated with spermatozoon may reflect the external exposure and internal body burden of environmental pollutants (Cai et al. 2015). The finding also echoes a recent proteomics study in which seminal proteins such as prosaposin may indicate the fertility ranking of the males in bulls (Viana et al. 2018).

A number of research groups have associated seminal plasma protein levels with semen parameters (Cao et al. 2018; Davalieva et al. 2012; Diamandis et al. 1999; Drabovich et al. 2013; Freour et al. 2013; Rolland et al. 2013; Wang et al. 2009). Panels of candidate proteins for male 
209

210

211

212

213

214

215

216

217

218

219

220

221

222

223

224

225

226

227

228

229

fertility have been proposed in studies comparing the proteomics data between normozoospermic

men and men with asthenozoospermia, oligozoospermia, or azoospermia. The semen parameters,

however, may only have limited predicting value for clinic outcomes among IVF patients.

Bartolacci et al. suggested that oligozoospermia according to WHO criteria may affect the embryo development but not top quality blastocyst formation rate or the establishment of pregnancy in ICSI cycles (Bartolacci et al. 2018). In a study including 1280 IVF cycles, Mariappen et al. suggested that the semen parameters have an insignificant role to play in embryo quality and overall outcomes (Mariappen et al. 2018). Similarly, Capelouto et al. found a lack of association between semen parameters and live birth rate in frozen donor oocyte cycles(Capelouto et al. 2018). These studies may lie on the fact that several critical processes of natural conception are bypassed by the ART treatment and suggest that male fertility biomarkers screened according to semen parameters may not be as feasible as they were expected in ART populations. In the present study, the association between seminal protein and outcomes of IVF treatment was not only observed in the overall population but also in normozoospermic men. On the other hand, neither sperm concentration nor sperm motility was significantly associated with normal fertilization and pregnancy. The data supported the hypothesis that seminal proteins provide more information regarding fertility than routine semen analysis and suggested that male factor might still affect the reproductive outcome even though the semen parameters are considered as normal according to the established criteria.

28 Our data also provide a detailed look at the association male factor and embryo development by linking the individual embryo morphological parameters with semen parameters 
230 and seminal protein using GEE. As shown in Table 5, male factors have been associated with 231 cleavage events at early development, such as early cleavage on day 1 and cleavage rates on day

232 3. Occurrence of early cleavage at a given time point is deemed as a significant predictor for embryo implantation (Alpha Scientists in Reproductive \& Embryology 2011). The use of modern time lapse technology further confirmed the importance of the timing and period of early cleavages before genomic activation in predicting embryo developmental competence (Kaser et al. 2017). Under experimental condition, it is shown that the $2^{\text {nd }}$ to $3^{\text {rd }}$ mitoses were sensitive periods in the presence of spermatozoal oxidative stress (Burruel et al. 2014). It could also be postulated that early cleavage events of embryos were also sensitive to spermatozoal oxidative stress derived from physiological or pathological conditions.

Due to the complexity of reproductive process, it is difficult to associate a single semen maker with pregnancy outcome. Conflicting evidence regarding the association between male factors and ART outcomes was not only observed in studied using semen parameters as male fertility markers(Bartolacci et al. 2018; Borges et al. 2016; Capelouto et al. 2018; Chapuis et al. 2017; Mariappen et al. 2018; Mazzilli et al. 2017), but also in those using other male fertility biomarkers, such as DNA fragmentation (Colaco \& Sakkas 2018). While many of the previous analyses were univariate in nature, the conflicting results may imply the importance of adjustment for the confounding factors associated with maternal factors. During the reproductive process, the female contribute to not only the maternal genetic material but also most of the cell machinery of the zygote and the environment for embryo implantation and fetal growth. In predicting the outcomes of IVF, maternal factors such as gynecological etiologies, ovarian 
251 response, maternal age and endometrial thickness may play significant roles (McLernon et al. 252 2016). In their study investigating the effects of male factor on ART outcomes, Mariappen et al. 253 found that female age but not male age or semen parameters has significant influence on 254 pregnancy or live birth (Mariappen et al. 2018). In our study, the association between male 255 fertility and IVF outcomes is strengthened by a prospective cohort in which female counterparts 256 with good prognosis were selected and important covariates for IVF outcomes were considered.

\section{Conclusions}

258 In conclusion, the present study demonstrated the association between prosaposin, a seminal secretory protein and the occurrence of fertilization and embryo cleavage events. Our data also suggested that seminal proteins may provide more information regarding IVF outcomes than traditional semen parameter could yield. Although the AUC suggest only a limited discriminating capacity of prosaposin to predict low fertilization events, a panel of seminal proteomics markers may provide a higher discriminating capacity in the future. 
265 Acknowledgments

266 The authors thank all the staff, especially the embryologists in our lab for their support in

267 generating this manuscript. We would like to thank Xinli Wang for her assistance in data 268 processing. 
270

271

272

273

274

275

276

277

278

279

280

281

282

283

284

285

286

287

288

289

290

291

292

293

294

295

296

297

298

299

300

301

302

303

304

305

306

307

308

309

References

Alpha Scientists in Reproductive M, and Embryology ESIGo. 2011. The Istanbul consensus workshop on embryo assessment: proceedings of an expert meeting. Hum Reprod 26:1270-1283. 10.1093/humrep/der037

Amann RP, Hammerstedt RH, and Shabanowitz RB. 1999a. Exposure of human, boar, or bull sperm to a synthetic peptide increases binding to an egg-membrane substrate. J Androl 20:34-41.

Amann RP, Seidel GE, Jr., and Brink ZA. 1999b. Exposure of thawed frozen bull sperm to a synthetic peptide before artificial insemination increases fertility. $J$ Androl 20:42-46.

Amann RP, Shabanowitz RB, Huszar G, and Broder SJ. 1999c. Increased in vitro binding of fresh and frozenthawed human sperm exposed to a synthetic peptide. J Androl 20:655-660.

Bartolacci A, Pagliardini L, Makieva S, Salonia A, Papaleo E, and Vigano P. 2018. Abnormal sperm concentration and motility as well as advanced paternal age compromise early embryonic development but not pregnancy outcomes: a retrospective study of 1266 ICSI cycles. J Assist Reprod Genet 35:1897-1903. 10.1007/s10815-018-1256-8

Bieniek JM, Drabovich AP, and Lo KC. 2016. Seminal biomarkers for the evaluation of male infertility. Asian $J$ Androl 18:426-433. 10.4103/1008-682X.175781

Borges E, Jr., Setti AS, Braga DP, Figueira RC, and Iaconelli A, Jr. 2016. Total motile sperm count has a superior predictive value over the WHO 2010 cut-off values for the outcomes of intracytoplasmic sperm injection cycles. Andrology 4:880-886. 10.1111/andr.12199

Burruel V, Klooster K, Barker CM, Pera RR, and Meyers S. 2014. Abnormal early cleavage events predict early embryo demise: sperm oxidative stress and early abnormal cleavage. Sci Rep 4:6598. 10.1038/srep06598

Cai JL, Sun LB, Guo ZZ, Jiang XM, Zheng GC, Qiu HL, Sha AG, Wang CG, Ren JZ, and Zuo ZH. 2015. Decrease in prosaposin in spermatozoon is associated with polychlorinated biphenyl exposure. Int J Clin Exp Pathol 8:2436-2448.

Cao X, Cui Y, Zhang X, Lou J, Zhou J, Bei H, and Wei R. 2018. Proteomic profile of human spermatozoa in healthy and asthenozoospermic individuals. Reprod Biol Endocrinol 16:16. 10.1186/s12958-018-0334-1

Capelouto SM, Nagy ZP, Shapiro DB, Archer SR, Ellis DP, Smith AK, Spencer JB, and Hipp HS. 2018. Impact of male partner characteristics and semen parameters on in vitro fertilization and obstetric outcomes in a frozen oocyte donor model. Fertil Steril 110:859-869. 10.1016/j.fertnstert.2018.06.003

Chapuis A, Gala A, Ferrieres-Hoa A, Mullet T, Bringer-Deutsch S, Vintejoux E, Torre A, and Hamamah S. 2017. Sperm quality and paternal age: effect on blastocyst formation and pregnancy rates. Basic Clin Androl 27:2. 10.1186/s12610-016-0045-4

Choy JT, and Eisenberg ML. 2018. Male infertility as a window to health. Fertil Steril 110:810-814. 10.1016/j.fertnstert.2018.08.015

Codognoto VM, Yamada PH, Schmith RA, de Ruediger FR, Scott C, de Faria Lainetti P, Brochine S, de Paula Freitas-Dell'Aqua C, de Souza FF, and Oba E. 2018. Functional insights into the role of seminal plasma proteins on sperm motility of buffalo. Anim Reprod Sci. 10.1016/j.anireprosci.2018.06.002

Colaco S, and Sakkas D. 2018. Paternal factors contributing to embryo quality. J Assist Reprod Genet 35:1953-1968. 10.1007/s10815-018-1304-4

Davalieva K, Kiprijanovska S, Noveski P, Plaseski T, Kocevska B, Broussard C, and Plaseska-Karanfilska D. 2012. Proteomic analysis of seminal plasma in men with different spermatogenic impairment. Andrologia 44:256- 
264. 10.1111/j.1439-0272.2012.01275.x

Diamandis EP, Arnett WP, Foussias G, Pappas H, Ghandi S, Melegos DN, Mullen B, Yu H, Srigley J, and Jarvi K. 1999. Seminal plasma biochemical markers and their association with semen analysis findings. Urology 53:596-603.

Drabovich AP, Dimitromanolakis A, Saraon P, Soosaipillai A, Batruch I, Mullen B, Jarvi K, and Diamandis EP. 2013. Differential diagnosis of azoospermia with proteomic biomarkers ECM1 and TEX101 quantified in seminal plasma. Sci Transl Med 5:212ra160. 10.1126/scitranslmed.3006260

ESHRE Special Interest Group of Embryology and Alpha Scientists in Reproductive Medicine. 2017. The Vienna consensus: report of an expert meeting on the development of ART laboratory performance indicators. Reprod Biomed Online 3 5: 494 -510.

Freour T, Com E, Barriere P, Bouchot O, Jean M, Masson D, and Pineau C. 2013. Comparative proteomic analysis coupled with conventional protein assay as a strategy to identify predictors of successful testicular sperm extraction in patients with non-obstructive azoospermia. Andrology 1:414-420. 10.1111/j.20472927.2012.00059.x

Hammerstedt RH, Cramer PG, Barbato GF, Amann RP, O'Brien JS, and Griswold MD. 2001. A fragment of prosaposin (SGP-1) from rooster sperm promotes sperm-egg binding and improves fertility in chickens. $J$ Androl 22:361-375.

Intasqui P, Antoniassi MP, Camargo M, Nichi M, Carvalho VM, Cardozo KH, Zylbersztejn DS, and Bertolla RP. 2015. Differences in the seminal plasma proteome are associated with oxidative stress levels in men with normal semen parameters. Fertil Steril 104:292-301. 10.1016/j.fertnstert.2015.04.037

Kaser DJ, Farland LV, Missmer SA, and Racowsky C. 2017. Prospective study of automated versus manual annotation of early time-lapse markers in the human preimplantation embryo. Hum Reprod 32:1604-1611. 10.1093/humrep/dex229

Magargee SF, Cramer PG, and Hammerstedt RH. 2000. Increased in vitro binding and fertilizing ability of mouse sperm exposed to a synthetic peptide. Mol Reprod Dev 57:406-411. 10.1002/10982795(200012)57:4<406::AID-MRD13>3.0.CO;2-8

Mariappen U, Keane KN, Hinchliffe PM, Dhaliwal SS, and Yovich JL. 2018. Neither male age nor semen parameters influence clinical pregnancy or live birth outcomes from IVF. Reprod Biol 18:324-329. 10.1016/j.repbio.2018.11.003

Mazzilli R, Cimadomo D, Vaiarelli A, Capalbo A, Dovere L, Alviggi E, Dusi L, Foresta C, Lombardo F, Lenzi A, Tournaye H, Alviggi C, Rienzi L, and Ubaldi FM. 2017. Effect of the male factor on the clinical outcome of intracytoplasmic sperm injection combined with preimplantation aneuploidy testing: observational longitudinal cohort study of 1,219 consecutive cycles. Fertil Steril 108:961-972 e963. 10.1016/j.fertnstert.2017.08.033

McLernon DJ, Steyerberg EW, Te Velde ER, Lee AJ, and Bhattacharya S. 2016. Predicting the chances of a live birth after one or more complete cycles of in vitro fertilisation: population based study of linked cycle data from 113873 women. BMJ 355:i5735. 10.1136/bmj.i5735

Morales CR, Hay N, El-Alfy M, and Zhao Q. 1998. Distribution of mouse sulfated glycoprotein-1 (prosaposin) in the testis and other tissues. $J$ Androl 19:156-164.

Ren J, Sha A, Han D, Li P, Geng J, and Ma C. 2014. Does prolonged pituitary down-regulation with gonadotropinreleasing hormone agonist improve the live-birth rate in in vitro fertilization treatment? Fertil Steril 
351

352

353

354

355

356

357

358

359

360

361

362

363

364

365

366

367

368

369

370

102:75-81. 10.1016/j.fertnstert.2014.03.030

Rolland AD, Lavigne R, Dauly C, Calvel P, Kervarrec C, Freour T, Evrard B, Rioux-Leclercq N, Auger J, and Pineau C. 2013. Identification of genital tract markers in the human seminal plasma using an integrative genomics approach. Hum Reprod 28:199-209. 10.1093/humrep/des360

Sakkas D, Ramalingam M, Garrido N, and Barratt CL. 2015. Sperm selection in natural conception: what can we learn from Mother Nature to improve assisted reproduction outcomes? Hum Reprod Update 21:711-726. 10.1093/humupd/dmv042

Sharma R, Agarwal A, Mohanty G, Jesudasan R, Gopalan B, Willard B, Yadav SP, and Sabanegh E. 2013. Functional proteomic analysis of seminal plasma proteins in men with various semen parameters. Reprod Biol Endocrinol 11:38. 10.1186/1477-7827-11-38

Viana AGA, Martins AMA, Pontes AH, Fontes W, Castro MS, Ricart CAO, Sousa MV, Kaya A, Topper E, Memili E, and Moura AA. 2018. Proteomic landscape of seminal plasma associated with dairy bull fertility. Sci Rep 8:16323. 10.1038/s41598-018-34152-w

Wang J, Wang J, Zhang HR, Shi HJ, Ma D, Zhao HX, Lin B, and Li RS. 2009. Proteomic analysis of seminal plasma from asthenozoospermia patients reveals proteins that affect oxidative stress responses and semen quality. Asian J Androl 11:484-491. 10.1038/aja.2009.26

WHO. 2010. WHO laboratory manual for the examination and processing of human semen. Geneva: World Health Organization. 


\section{Table $\mathbf{1}$ (on next page)}

Patient characteristics andcycle parameters 


\section{Table 1:}

\section{Patient characteristics and cycle parameters}

\begin{tabular}{|c|c|}
\hline $\mathrm{n}$ & 166 \\
\hline \multicolumn{2}{|l|}{ Female characteristics } \\
\hline Female age, year & $31[5]$ \\
\hline $\mathrm{BMI}, \mathrm{kg} / \mathrm{m}^{2}$ & $21.3[3.23]$ \\
\hline Basal FSH, mIU/ml & $6.66[2.17]$ \\
\hline Basal LH, mIU/ml & $4.03[2.12]$ \\
\hline Basal E2, pg/ml & $35[25.25]$ \\
\hline $\mathrm{AFC}$ & $12[7]$ \\
\hline \multicolumn{2}{|l|}{ Male characteristics } \\
\hline Male age, year & $32[6.25]$ \\
\hline BMI, $\mathrm{kg} / \mathrm{m}^{2}$ & $23.51[4.36]$ \\
\hline Smoker (\%) & $94(56.6)$ \\
\hline Prosaposin, $\mathrm{ng} / \mathrm{ml}$ & $73.12[95.52]$ \\
\hline Semen volume, ml & $2.5[1]$ \\
\hline $\mathrm{FSH}, \mathrm{mIU} / \mathrm{ml}$ & $5.15[1.91]$ \\
\hline Sperm concentration, $\times 10^{6} / \mathrm{ml}$ & $59.69[56.2]$ \\
\hline Total sperm count, $\times 10^{6}$ & $149.96[154.16]$ \\
\hline Normal morphology, $\%$ & $6.5[6]$ \\
\hline Motility, \% & $50.35[22.37]$ \\
\hline Total motile sperm, $\times 10^{6}$ & $68.96[84.13]$ \\
\hline Progressive, $\%$ & $36.74[18]$ \\
\hline Non progressive, $\%$ & $9[7.02]$ \\
\hline Immotile, \% & $49.15[22.06]$ \\
\hline Postwash sperm concentration, $\times 10^{6} / \mathrm{ml}$ & $40[15]$ \\
\hline Postwash sperm motility, \% & $98[3]$ \\
\hline Postwash progressive motility, $\%$ & $95[5]$ \\
\hline Postwash nonprogressive motility, $\%$ & $3[2]$ \\
\hline \multicolumn{2}{|l|}{ Cycle parameters } \\
\hline Gonadotropin dose, IU & $2475[740.63]$ \\
\hline Duration of stimulation, day & $12[3]$ \\
\hline Starting dose, IU & $225[75]$ \\
\hline Oocyte yield & $11[4]$ \\
\hline Endometrial thickness, mm & $10.9[3.45]$ \\
\hline Fertilization rate, $\%$ & $87.87[22.22]$ \\
\hline Normal fertilization rate, $\%$ & $70[22.86]$ \\
\hline Normal fertilization $<50 \%(\%)$ & $29(17.5)$ \\
\hline Good embryo proportion, $\%$ & $63.07[35.12]$ \\
\hline
\end{tabular}


ET cancelled (\%)

Cleavage ET (\%)

Blastocyst ET (\%)

Number of embryos transferred

Pregnancy/ET (\%)
$7(4.2)$

$111(69.8)$

$48(30.2)$

2 [1]

103/159 (64.78)

3 ET, embryo transfer; Data are median [IQR] or count (percentage). 
Table 2 (on next page)

Correlationbetween prosaposin concentration and semen parameters 
1 Table 2:

2 Correlation between prosaposin concentration and semen parameters

\begin{tabular}{lcc}
\hline & $\begin{array}{c}\text { Correlation } \\
\text { coefficient }\end{array}$ & $\mathrm{P}$ \\
\hline Sperm concentration & 0.13 & 0.096 \\
Normal morphology & -0.153 & 0.051 \\
Motility & -0.01 & 0.893 \\
Progressive motility & 0.197 & 0.011 \\
Postwash sperm concentration & -0.115 & 0.141 \\
Postwash sperm motility & 0.269 & $<0.001$ \\
Postwash progressive motility & 0.266 & 0.001 \\
FSH levels & 0.098 & 0.21 \\
\hline
\end{tabular}

3 


\section{Table 3(on next page)}

Multivariateanalysis for normal fertilization rate and good embryo proportion, with respectto prosaposin concentration and semen quality

Each model was adjusted for age of the couple, female BMI, basal FSH, AFC, starting dose, oocyte yield and male smoker 
1 Table 3:

2 Multivariate analysis for normal fertilization rate and good embryo proportion, with respect to prosaposin

3 concentration and semen quality.

\begin{tabular}{lcccc}
\hline & \multicolumn{2}{c}{ Normal fertilization } & \multicolumn{2}{c}{ Good embryo proportion } \\
& Coefficient & $\mathrm{P}$ & Coefficient & $\mathrm{P}$ \\
\hline Overall population, $\mathrm{n}=166$ & & & & \\
$\quad$ Prosaposin & 0.066 & 0.005 & 0.070 & 0.038 \\
Sperm concentration & 0.046 & 0.118 & -0.040 & 0.342 \\
Male FSH & 0.169 & 0.801 & -1.984 & 0.033 \\
Normal morphology & 0.115 & 0.685 & 0.100 & 0.800 \\
Motility & 0.007 & 0.934 & -0.203 & 0.422 \\
Progressive motility & 0.067 & 0.499 & 0.067 & 0.632 \\
Postwash sperm concentration & -0.071 & 0.554 & -0.273 & 0.101 \\
Postwash motility & 0.005 & 0.979 & -0.234 & 0.358 \\
Postwash progressive motility & 0.077 & 0.664 & -0.210 & 0.399 \\
Normozoospermia, $\mathrm{n}=87$ & & & & \\
Prosaposin & 0.066 & 0.042 & 0.049 & 0.325 \\
Sperm concentration & 0.045 & 0.330 & 0.006 & 0.925 \\
Male FSH & -0.248 & 0.781 & -2.3 & 0.066 \\
Normal morphology & 0.233 & 0.577 & 0.314 & 0.595 \\
Motility & -0.038 & 0.837 & -0.059 & 0.819 \\
Progressive motility & 0.026 & 0.921 & 0.244 & 0.511 \\
Postwash sperm concentration & 0.194 & 0.296 & -0.479 & 0.066 \\
Postwash motility & 0.855 & 0.586 & 4.681 & 0.032 \\
Postwash progressive motility & 0.843 & 0.361 & 1.940 & 0.135 \\
\hline
\end{tabular}

4 Each model was adjusted for age of the couple, female BMI, female basal FSH, AFC, starting dose,

5 oocyte yield and male smoker

6 


\section{Table 4 (on next page)}

Discriminatingcapacity of prosaposin concentration and semen quality for low fertilization(normal fertilization $<50 \%$ ) in ROC curves. 
1 Table 4:

2 Discriminating capacity of prosaposin concentration and semen quality for low fertilization (normal

3 fertilization $<50 \%$ ) in ROC curves.

\begin{tabular}{lccccc}
\hline & Cutoff & Sensitivity & Specificity & AUC $(95 \% \mathrm{CI})$ & $\mathrm{P}$ \\
\hline Overall population, $\mathrm{n}=166$ & & & & & \\
Prosaposin & 63.35 & 0.76 & 0.60 & $0.666(0.569-0.763)$ & 0.006 \\
Sperm concentration & 48.63 & 0.55 & 0.67 & $0.582(0.46-0.705)$ & 0.171 \\
FSH & 3.39 & 0.86 & 0.21 & $0.474(0.362-0.585)$ & 0.655 \\
Normal morphology & 17.40 & 1.00 & 0.07 & $0.433(0.325-0.541)$ & 0.266 \\
Motility & 63.53 & 0.93 & 0.15 & $0.511(0.389-0.632)$ & 0.861 \\
Progressive motility & 36.74 & 0.59 & 0.53 & $0.548(0.426-0.67)$ & 0.429 \\
Postwash sperm concentration & 52.50 & 0.90 & 0.19 & $0.529(0.419-0.638)$ & 0.632 \\
Postwash motility & 96.50 & 0.48 & 0.72 & $0.604(0.483-0.725)$ & 0.085 \\
Postwash progressive motility & 91.00 & 0.48 & 0.69 & $0.602(0.485-0.719)$ & 0.089 \\
Normozoospermia, $\mathrm{n}=87$ & & & & & \\
Prosaposin & 63.35 & 0.88 & 0.62 & $0.707(0.592-0.822)$ & 0.010 \\
Sperm concentration & 48.63 & 0.50 & 0.77 & $0.61(0.456-0.764)$ & 0.171 \\
FSH & 8.81 & 0.063 & 0.972 & $0.436(0.284-0.588)$ & 0.436 \\
Normal morphology & 11.75 & 0.88 & 0.25 & $0.437(0.294-0.581)$ & 0.437 \\
Motility & 63.53 & 0.88 & 0.21 & $0.412(0.248-0.576)$ & 0.276 \\
Progressive motility & 36.74 & 0.25 & 0.87 & $0.516(0.346-0.686)$ & 0.844 \\
Postwash sperm concentration & 31.50 & 0.38 & 0.86 & $0.614(0.446-0.781)$ & 0.158 \\
Postwash motility & 96.00 & 0.38 & 0.82 & $0.547(0.372-0.722)$ & 0.558 \\
Postwash progressive motility & 91.00 & 0.38 & 0.79 & $0.573(0.414-0.732)$ & 0.363 \\
\hline
\end{tabular}

4 


\section{Table 5 (on next page)}

Associationbetween embryo parameters and prosaposin concentration/semen quality 
1 Table 5:

2 Association between embryo parameters and prosaposin concentration/semen quality.

\begin{tabular}{|c|c|c|c|c|c|c|c|}
\hline & Early cleavage on day 1 & $\begin{array}{l}\text { Fast cleaving embryo on } \\
\text { day3 }\end{array}$ & $\begin{array}{c}\text { Slow cleaving embryo } \\
\text { on day } 3\end{array}$ & $\begin{array}{c}\text { On time } 8 \text { cell embryo } \\
\text { on day } 3\end{array}$ & $\begin{array}{l}\text { Unsynchronized } \\
\text { cleaving embryo on } \\
\text { day3 }\end{array}$ & $\begin{array}{l}\text { Fragmentation }>10 \% \text { on } \\
\text { day } 3\end{array}$ & $\begin{array}{l}\text { Uneven cl } \\
\text { embryo ol }\end{array}$ \\
\hline \multicolumn{8}{|l|}{ Overall population, $\mathrm{n}=1252$} \\
\hline Prosaposin & $1.005(1.002-1.009)^{* *}$ & $1.002(0.999-1.005)$ & $0.999(0.996-1.002)$ & $0.999(0.997-1.002)$ & $1.001(0.999-1.003)$ & $0.997(0.993-1.001)$ & $0.997(0.995$ \\
\hline Sperm concentration & $1.001(0.997-1.005)$ & $0.998(0.994-1.002)$ & $1.002(0.997-1.006)$ & $1.001(0.997-1.003)$ & $1.001(0.998-1.002)$ & $1.001(0.995-1.004)$ & $1.001(0.998$ \\
\hline Male FSH & $0.962(0.89-1.04)$ & $1.051(0.98-1.128)$ & $0.99(0.94-1.045)$ & $0.969(0.93-1.014)$ & $0.978(0.93-1.024)$ & $1.043(0.96-1.137)$ & $1.07(1.01-$ \\
\hline Normal morphology & $0.994(0.952-1.038)$ & $0.995(0.963-1.029)$ & $0.992(0.966-1.018)$ & $1.015(0.993-1.037)$ & $0.997(0.977-1.017)$ & $0.981(0.933-1.033)$ & $0.984(0.952$ \\
\hline Motility & $0.999(0.986-1.011)$ & $0.998(0.979-1.017)$ & $0.994(0.98-1.008)$ & $1.002(0.989-1.016)$ & $0.999(0.992-1.006)$ & $0.96(0.876-1.053)$ & $1.011(0.99$ \\
\hline Progressive motility & $1.020(1.005-1.036)^{* *}$ & $0.992(0.981-1.003)$ & $1.001(0.988-1.012)$ & $1.002(0.992-1.012)$ & $1.003(0.993-1.013)$ & $1.012(0.994-1.030)$ & $1.003(0.992$ \\
\hline Postwash sperm concentration & $0.997(0.982-1.013)$ & $1.001(0.987-1.014)$ & $1.003(0.992-1.014)$ & $0.996(0.986-1.006)$ & $1.007(0.999-1.016)$ & $1.015(0.953-1.081)$ & $1.003(0.986$ \\
\hline Postwash motility & $1.087(0.960-1.232)$ & $0.982(0.974-0.991) * *$ & $1.007(0.999-1.015)$ & $1.012(0.994-1.030)$ & $1.005(0.998-1.013)$ & $1.016(0.954-1.081)$ & $1.020(0.98$ \\
\hline Postwash progressive motility & $1.028(0.983-1.076)$ & $0.980(0.968-0.991) * *$ & $1.010(0.999-1.020)$ & $1.010(0.994-1.026)$ & $1.003(0.993-1.014)$ & $1.017(0.955-1.082)$ & $1.008(0.98$ \\
\hline \multicolumn{8}{|l|}{ Normozoospermia, $\mathrm{n}=645$} \\
\hline Prosaposin & $1.003(0.999-1.007)$ & $1.004(1.001-1.007)^{* *}$ & $0.996(0.993-0.999)^{*}$ & $1.001(0.998-1.002)$ & $1.001(0.998-1.003)$ & $0.997(0.991-1.003)$ & $1.001(0.99$ \\
\hline Sperm concentration & $1.003(0.998-1.008)$ & $1(0.996-1.005)$ & $0.999(0.995-1.003)$ & $1.002(0.999-1.006)$ & $0.998(0.994-1.002)$ & $0.996(0.987-1.005)$ & $0.998(0.992$ \\
\hline Male FSH & $0.936(0.88-0.996)^{*}$ & $0.96(0.89-1.039)$ & $1.061(1-1.127)$ & $0.964(0.92-1.009)$ & $0.996(0.95-1.05)$ & $1.077(0.94-1.23)$ & $1(0.94-1$ \\
\hline Normal morphology & $0.998(0.933-1.067)$ & $1.024(0.974-1.076)$ & $0.99(0.948-1.034)$ & $1.003(0.974-1.033)$ & $1.019(0.99-1.049)$ & $0.939(0.88-1.001)$ & $0.999(0.96$ \\
\hline Motility & $0.995(0.974-1.017)$ & $1.009(0.991-1.028)$ & $0.987(0.968-1.006)$ & $1.007(0.993-1.022)$ & $0.987(0.969-1.004)$ & $0.98(0.945-1.017)$ & $0.985(0.966$ \\
\hline Progressive motility & $1.019(0.991-1.048)$ & $0.992(0.968-1.017)$ & $0.983(0.959-1.006)$ & $1.021(1.003-1.039)^{*}$ & $0.995(0.973-1.017)$ & $1.003(0.963-1.045)$ & $0.991(0.966$ \\
\hline Postwash sperm concentration & $1.003(0.982-1.025)$ & $1.001(0.98-1.022)$ & $1.019(0.998-1.04)$ & $0.984(0.97-0.998)^{*}$ & $1.016(1.00-1.033)^{*}$ & $1.031(0.997-1.066)$ & $1.004(0.98$ \\
\hline Postwash motility & $1.017(0.811-1.275)$ & $1.022(0.847-1.234)$ & $0.935(0.793-1.101)$ & $1.062(0.924-1.22)$ & $0.943(0.831-1.07)$ & $0.896(0.696-1.155)$ & $0.978(0.83$ \\
\hline Postwash progressive motility & $0.968(0.846-1.107)$ & $0.985(0.883-1.099)$ & $1.011(0.914-1.119)$ & $1.011(0.934-1.093)$ & $0.977(0.906-1.053)$ & $0.949(0.82-1.098)$ & $0.966(0.878$ \\
\hline
\end{tabular}

3 Each model was adjusted for age of the couple, female BMI, basal FSH, AFC, starting dose, oocyte yield and male smoker 
4 Fast cleaving embryo was defined as embryo with more than 8 cells at the time of observation; Slow cleaving embryo was defined as embryo with less than 8

5 cells at the time of observation; Nonsynchronized cleaving embryo was defined as embryo with even cell number at the time of observation.

6 “*” indicates significant at $\mathrm{P}<0.05$ 


\section{Table 6 (on next page)}

Multivariate analysis forclinical pregnancy $(n=157)$ 
1 Table 6:

2 Multivariate analysis for clinical pregnancy $(n=157)$

\begin{tabular}{|c|c|c|c|}
\hline \multirow[t]{2}{*}{ Variable } & \multirow[t]{2}{*}{ Category } & \multicolumn{2}{|c|}{ OR $(95 \% \mathrm{CI})$} \\
\hline & & Overall & Normozoospermia, \\
\hline Female age & per year increased & $0.973(0.834-1.135)$ & $0.905(0.704-1.162)$ \\
\hline Male age & per year increased & $1.028(0.899-1.175)$ & $1.095(0.885-1.355)$ \\
\hline Female BMI & per unit increased & $0.982(0.859-1.122)$ & $0.917(0.768-1.094)$ \\
\hline Female basal FSH & per $\mathrm{mIU} / \mathrm{ml}$ increased & $0.985(0.802-1.211)$ & $1.559(0.992-2.451)$ \\
\hline Male FSH & per $\mathrm{mIU} / \mathrm{ml}$ increased & $1.06(0.877-1.281)$ & $1.041(0.718-1.507)$ \\
\hline $\mathrm{AFC}$ & per AFC increased & $1.008(0.923-1.101)$ & $1.067(0.933-1.219)$ \\
\hline Male smoker & no smoker vs smoker & $0.468(0.206-1.063)$ & $0.597(0.158-2.249)$ \\
\hline Prosaposin concentration & per $\mathrm{ng} / \mathrm{ml}$ increased & $1.009(1.001-1.016)^{*}$ & $1.018(1.004-1.032)^{*}$ \\
\hline Semen volume & per $\mathrm{ml}$ increased & $1.687(1.059-2.687)^{*}$ & $3.3(1.448-7.52)^{*}$ \\
\hline Sperm concentration & per $10^{6} / \mathrm{ml}$ increased & $0.994(0.986-1.003)$ & $0.991(0.976-1.006)$ \\
\hline Normal morphology & per percentage increased & $1.019(0.935-1.11)$ & $1.258(1.048-1.511)^{*}$ \\
\hline Motility & per percentage increased & $1.007(0.984-1.031)$ & $1.029(0.973-1.087)$ \\
\hline Starting dose & per IU increased & $1.002(0.991-1.015)$ & $1.01(0.991-1.029)$ \\
\hline Oocyte yield & per oocyte increased & $1.094(0.956-1.252)$ & $1.121(0.881-1.426)$ \\
\hline Distance to fundal & per $\mathrm{cm}$ increased & $0.953(0.308-2.953)$ & $0.303(0.046-1.985)$ \\
\hline Number of embryo transferred & per embryo increased & $0.697(0.219-2.219)$ & $4.761(0.452-50.106)$ \\
\hline Endometrial thickness & per mm increased & $1.244(1.036-1.493)^{*}$ & $1.221(0.932-1.6)$ \\
\hline At least on top quality embryo transferred & yes vs no & $5.541(1.436-21.381)^{*}$ & $35.2(2.846-435.317)^{*}$ \\
\hline Stage of embryo transfer & cleavage vs blastocyst & $1.158(0.334-4.021)$ & $8.031(0.704-91.582)$ \\
\hline
\end{tabular}

3

4 “*” indicates significant at $\mathrm{P}<0.05$ 